Meta

Journal des traducteurs

Translators' Journal

\title{
Littérature et titres de presse : le cas italien
}

\section{Michel Bastiaensen}

Volume 39, numéro 1, mars 1994

La traduction et l'interprétation dans la Belgique multilingue

URI : https://id.erudit.org/iderudit/001981ar

DOI : https://doi.org/10.7202/001981ar

Aller au sommaire du numéro

Éditeur(s)

Les Presses de l'Université de Montréal

ISSN

0026-0452 (imprimé)

1492-1421 (numérique)

Découvrir la revue

Citer cet article

Bastiaensen, M. (1994). Littérature et titres de presse : le cas italien. Meta, 39(1),

229-240. https://doi.org/10.7202/001981ar d'utilisation que vous pouvez consulter en ligne.

https://apropos.erudit.org/fr/usagers/politique-dutilisation/ 


\title{
LITTÉRATURE ET TITRES DE PRESSE: LE CAS ITALIEN
}

\author{
MiCHEL BASTIAENSEN \\ ESTI (Institut Cooremans), ULB, VUB, Belgique
}

Le titre est une réalité multiple, à la fois étiquette, aperçu du contenu et slogan, conformément à ses fonctions appellative, référentielle et conative ( $c f$. Jacques in HallynJacques 1987: 205). Ses différentes implications linguistiques, sémiotiques, sigmatiques, pragmatiques, etc. sont assez vastes et complexes pour avoir donné lieu à une microdiscipline dénommée «titrologie», dont la bibliographie commence à être assez fournie (cf. Hoek 1981 : 339-349, etc.; Genette 1987: 54, note 1). Le titre est aussi le lieu (privilégié puisqu'il vient en tête du texte) où s'exercent différents effets de langue et de style: recherche de la concision et de l'expressivité, souci du rythme et de l'euphonie, artifices symboliques et rhétoriques, «jeux» divers de fond et de forme; autant de traits qui transcendent sa fonction purement informative. Il en est ainsi des titres des livres ( UUn beau titre est le vrai proxénète d'un livre», Furetière in Genette 1987:87) et des titres d'articles de presse - les deux variétés gardant, à de nombreux autres égards, leur caractère propre.

Parmi ces artifices, l'intertextualité occupe une place non négligeable (Hoek 1981: 183-199, parle à ce propos d' «intertitularité»). Nombreuses sont les références, plus ou moins explicites et perceptibles, à un ensemble de textes préexistants, à structure fortement stéréotypée, communs à l'auteur et au lecteur: proverbes, expressions idiomatiques, techniques, bureaucratiques, etc.; Sandulescu (1986:609) les appelle archetypes et paragonclichés, et il y voit quelque analogie avec la dichotomie type / token.

Quel que soit le nom qu'on leur donne, le fait est qu'un certain nombre de ces stéréotypes appartiennent au domaine littéraire, au sens le plus large du mot: grandes œuvres classiques, romans policiers, poésies, livres «utilitaires», ouvrages pour la jeunesse, maximes, paroles célèbres, contes, chansons, bandes dessinées: textes autochtones et étrangers, anciens et modernes, de valeur très inégale, fruits d'une connaissance directe ou d'ouï-dire, etc.

Point n'est besoin de souligner l'importance de ce phénomène pour la compréhension d'un texte dans sa totalité, ni d'insister sur la nécessité, pour quiconque s'occupe de la langue - apprentissage, enseignement, traduction - de savoir reconnaître et situer ce processus intertextuel particulier.

Dans les pages qui suivent, nous nous proposons d'examiner de plus près ces réminiscences littéraires telles qu'elles apparaissent dans les titres d'articles de presse ${ }^{1}$ en Italie, en nous appuyant sur un corpus d'environ 450 cas, fournis par les hebdomadaires L'Espresso (abrégé: E) et Panorama (abrégé : P) entre 1986 et les premiers mois de 1993 (le dépouillement ne prétend pas à l'exhaustivité).

Ces titres d'articles qui se parent de souvenirs littéraires avaient déjà retenu notre attention il y a une douzaine d'années, donnant lieu à une enquête sur environ 300 cas, à partir, essentiellement, de E, entre 1974 et 1981 (Bastiaensen 1982a). Ce premier corpus n'apparaîtra ici que comme terme de comparaison. Il nous avait semblé, à l'époque, que cette «intertextualité littéraire» s'exerçait avec une force toute particulière dans le domaine italien, ce que confirma, a contrario, une étude parallèle menée sur des hebdomadaires 
français (Bastiaensen 1982b): nous n'oserions pourtant plus être aussi catégorique aujourd'hui ${ }^{2}$. Nous ne saurions toutefois réexaminer la question dans les limites de cet article, ni en aborder d'autres, comme la proportion quantitative entre «titres intertextuels» et «non intertextuels», à intertexte littéraire ou non, leur récurrence selon les époques, leur rapport avec le niveau culturel et le prestige social des hebdomadaires envisagés; etc. De même, les contraintes d'espace ne nous permettront pas de faire le tour complet de la question, ni de multiplier les exemples, ni d'analyser chacun comme il le mériterait.

Deux problèmes se sont posés d'emblée lors de la constitution du corpus : les limites du concept «littérature» et l'identification des références intertextuelles. Le premier consistait à savoir s'il fallait aussi inclure dans ce secteur littéraire les allusions au cinéma. S'agissant, dans un cas comme dans l'autre, de moyens d'expression utilisant du moins en partie le même système modélisant secondaire, nous serions tenté de répondre par l'affirmative. D'autre part, si on n'a aucune hésitation à faire rentrer parmi les œuvres littéraires les pièces de théâtre, il n'y a pas, a priori, de raisons d'exclure les films. En fait, les relations entre cinéma et littérature nous paraissent trop étroites pour que nous décidions - arbitrairement — d'éliminer les allusions aux œuvres du septième art. Par ailleurs, cette option n'enlève rien au caractère spécifique des messages cinématographiques, qui occupent sans conteste une place à part au sein de ces «réminiscences littéraires» au sens large, ce qui a pu justifier qu'on ait consacré récemment une étude spéciale aux titres de films dans la langue commune (Medici et Cappelluzzo Springolo 1991).

Le second problème préliminaire peut être formulé en ces termes: quand et comment avoir la certitude qu'un titre se réfère à un intertexte littéraire précis? Il ne suffit pas de trouver un titre comme Les chaises (le cas est inventé) pour être en droit de conclure automatiquement à une réminiscence d'Ionesco. À plus forte raison en est-il ainsi lorsque les segments ne sont pas identiques : rien ne permet de voir dans Elogio della volgarità (P 20-XII-92) et Elogio della fluttuazione (E 18 X 92) une référence à l'Éloge de la Folie d'Érasme ou l'Elogio degli uccelli de Leopardi, ni dans Maledetto sia il Bot (E 7 II 93) un rappel de la Bible ou d'un sonnet (LI) de Cecco Angiolieri. En fait, de tels titres sont totalement atypiques. Il est difficile de donner une règle absolue, et sans doute la subjectivité y a-t-elle sa part. On peut raisonnablement supposer qu'il y a, parmi les «indices d'intertextualité», des phénomènes tels que la présence dans le titre de mots statistiquement rares (untore ne peut qu'évoquer Manzoni), ainsi que de noms propres personnels (Galeotto ne peut renvoyer qu'à Dante), de mots d'une autre langue ([x] mon amour, Françoise Sagan; De bello [x], César), de constructions et de figures de style insolites (l'hypallage il silenzio verde, Carducci), d'archaïsmes (vo pour vado; mots «tronqués», cf. altar, etc.); des faits de syntaxe, de morphologie, d'accentuation, de rythme interviennent également. Il est probable que la présence simultanée de plusieurs de ces faits rendra plus forte la présomption qu'il y a réminiscence littéraire et que le degré de certitude de cette présomption croîtra en fonction de la quantité d'information donnée par des éléments du titre (information signifiant ici non pas «ce que chaque mot apprend en moyenne», mais «mesure [...] de l'élément de surprise, fructueuse ou non, qu'apporte en moyenne la réception de chaque nouveau mot» (Mandelbrot 1954: 11-12).

Une fois reconnus avec un degré suffisant de certitude ces titres empruntés à la littérature, on pourrait, d'une manière tout empirique, les répartir selon la double opposition: «littéral» $v s$ «non littéral» et «ouvert» vs «fermé». En effet, les citations, qu'elles soient littérales ou modifiées, peuvent prendre place dans un titre à séquence «fermée», semblable à celle de la citation de départ, dans son ampleur comme dans sa structure. Elles peuvent aussi entrer dans une séquence «ouverte», où elles sont intégrées dans une structure différente et/ou dans un contexte plus vaste ou moins vaste, parfois jusqu'à s'y diluer. Concrètement, une séquence sera dite «ouverte» quand des éléments ont été ajoutés ou 
supprimés au début, à la fin ou au milieu de la citation, littérale ou non. On obtient ainsi quatre types:

Type I : Littéral, fermé.

Ex.: Lavorare stanca (E 18 X 87 ; Pavese)

Type II : Non littéral, fermé.

Ex.: Lottizzare stanca (P 8 XI 92; Pavese)

Type III : Littéral, ouvert.

Ex.: Va' pensiero, si, ma dove? (E 21 IX 86; VerdiSolera)

Type IV : Non littéral, ouvert. Ex. : Il silenzio è verde (cf. Carducci : «Il divino del pian silenzio verde $\left.{ }^{3} \gg\right)$.

Nous nous limiterons dans cet article à l'examen des deux séquences «fermées», Type I et Type II.

\section{TYPE I : LITTÉRAL, FERMÉ}

Qu'est-ce qui peut motiver, au-dessus d'un article de presse, la présence d'une citation littérale prise à un autre texte? Pour qu'un tel titre ne paraisse pas gratuit, il faut qu'il ait un «ancrage» dans le co-texte ${ }^{4}$, en vertu d'une nécessaire relation de «dépendance contextuelle» réciproque (Hoek 1981: 152). Pour que ce genre de relation soit possible dans le cas qui nous occupe, il faut bien qu'il y ait dans le texte cité un élément sémantique commun au co-texte, auquel il va servir de titre, par-delà les différences des deux textes de départ. Un tel transfert d'un ensemble signifiant n'est pas sans rappeler le mécanisme des figures de pensée. Et, de fait, dans l'applicabilité de la citation à un co-texte différent, on pourrait distinguer trois procédés, qui rappellent la métonymie, la métaphore et la synecdoque - à condition de ne pas utiliser ces termes de manière trop restrictive, d'être conscient qu'il peut y avoir des chevauchements et des cas douteux, et que plusieurs citations identiques peuvent relever, selon les co-textes auxquels ils s'appliquent, de figures différentes.

a) Synecdoque. Celle-ci opérant «un changement dans l'extension logique du mot», substituant «à un mot un terme d'extension différente» (Henry 1984:20), on peut considérer comme relevant de ce procédé les cas où la citation coiffe un nouveau co-texte en vertu d'une extension ou d'une restriction du champ d'application. P. ex. Giacomo il Fatalista (E $25 \times$ 87), traduction de Jacques le Fataliste, peut s'appliquer à «tous les Jacques qui sont fatalistes", donc aussi à un Jacques particulier, Giacomo Leopardi; Eppur si muove (E 20 IV 86), attribué à Galilée, peut s'étendre à «tout ce qui bouge», donc aussi à la dérive des continents; $C^{\prime}$ eravamo tanto amati $(\mathrm{E} 11 \mathrm{X} 87)$ à tout texte où il est question d'amoureux; et intituler un article sur les problèmes écologiques de Florence La notte di San Lorenzo (E 13 VIII 89) revient à désigner le tout par la partie.

b) Métonymie. C'est à une démarche métonymique - opérant sur la compréhension logique (Henry 1984: 20) — qu'on peut ramener les titres où la citation littérale s'accompagne d'un passage du concret à l'abstrait et vice versa. $\mathrm{P}$. ex. : Il gran rifiuto $(\mathrm{E}, 1986)$, souvenir de Dante (Inf. III, 60), qui parle probablement de Célestin V abdiquant la papauté, est ici appliqué au problème des déchets (rifiuto=, aussi, «déchet»); de même Le luci della ribalta (E 11 X 87), «les feux de la rampe» évoquant le monde du spectacle dans ses aspects les plus chatoyants, se réfère aux fouilles archéologiques dans le théâtre grec de Taormina. On pourra aussi ranger ici, malgré la suppression de la particule vocative $o$ : T'amo, pio bove (E 7 III 93), qui se réfère à rien de moins que la zoophilie ! Quant aux célèbres expressions métaphoriques Oppio del popolo (E 16 X 88: sur le problème de la drogue) et Via col vento (E 6 VII 86: les énergies alternatives), elles reviennent à leurs racines concrètes et ressortissent donc aussi à la métonymie. 
c. Métaphore. Le recours à la métaphore est relativement rare dans notre corpus. On trouve néanmoins Paradiso perduto (E 14 I 90) et Paradiso ritrovato (P 10 III 91), deux rappels miltoniens se référant l'un à Madagascar, «île de vacances à risques», l'autre à l'Italie, qui voit se reconstituer petit à petit sa faune sauvage. On peut aussi rattacher à la métaphore les animaux de la fable («Cet homme est un renard») dans La Volpe e il Corvo (P 19 VII 92), désignant MM. Andreotti et Sbardella.

Quelle que soit la figure qui lui permet de fonctionner, force est de constater que la citation littérale utilisée comme titre jouit d'une faveur plutôt modeste : 26 cas, soit 5,77\% du corpus. Assez rares, en effet, sont les cas où un texte peut entretenir une relation harmonieuse, non gratuite et non obscure, qui paraisse «nécessaire», avec un ensemble signifiant d'une tout autre provenance. Dans tous les cas, c'est le co-texte qui «explique» le titre, ce qui rappelle la référence anaphorique (Hoek $1981: 152,155)$.

\section{TYPE II : NON LITTÉRAL, FERMÉ}

La majeure partie de notre corpus (362 cas, $80,44 \%$ ) consiste en des citations «déformées», encore que facilement reconnaissables et réductibles à un modèle cité. Les écarts par rapport à celui-ci sont dus à des substitutions sur l'axe paradigmatique, lesquelles opèrent un raccord explicite avec le co-texte.

Ce raccord peut être obtenu par une substitution dans les morphèmes, les éléments grammaticaux et/ou les «mots vides»: permutations de nombre (Oltre gli specchi, P 31 I 93), de genre (Brutte, sporche e cattive, E 24 I 93), de personne (Le loro prigioni, E 21 VI 92; E 26 VII 92); adjonctions et suppressions de la négation (Eppur non si muove, E 2 VIII, 92; Le città visibili, E 15 XI 87). Toutes ces modifications réorientent le message du titre cité vers l'objet de l'article (arrestation de politiciens véreux, gangs de femmes, immobilisme...). Il n'est pas rare que les permutations portent sur les articles et autres déterminatifs (Un'opera aperta, E 20 VIII 89, sur une histoire de la littérature russe; Dolenti note, $\mathrm{P} 30$ VIII 92, sur les problèmes de la musique classique; 11 mio contesto, $\mathrm{E}$ 24 XII 89, interview de Sciascia), ce qui a «pour effet de donner au titre une fonction généralisante ou bien particularisante» (Hoek 1981: 365); bien que leurs modalités ne soient pas identiques en français et en italien, de telles permutations semblent en tout état de cause correspondre au refus de donner le contenu de l'article pour identique au texte de départ. Les prépositions peuvent elles aussi être modifiées, parfois avec des effets singulièrement efficaces, comme dans Tanto rumore nel nulla (E 1 III 87: c.r. du Livre de l'inquiétude de Pessoa), où la substitution suffit à donner à ce nulla une dimension métaphysique («néant») qu'il n'avait pas dans la comédie de Shakespeare («rien»).

Dans l'immense majorité des cas, toutefois, la substitution porte sur des éléments lexicaux: c'est là visiblement le procédé le plus aimé et le plus productif. L'abondance est d'ailleurs telle qu'il n'est pas facile d'y dégager autre chose que quelques lignes de force.

Un nombre limité de cas relèvent d'un mécanisme d'association, souvent l'opposition: Partire è un po' rinascere (P 17 I 92, sur l'kexil volontaire» de Paul Bowles); C'eravamo tanto odiati (E 23 VI 91); Parigi o amara (P 24 II 91). Exceptionnellement, l'opposition «sur l'axe vertical» se double d'une autre, «sur l'axe horizontal»: Divino, troppo umano (E 23 XI 86, c.r. d'un livre sur Jésus). Ailleurs, on fait appel à la similarité, qui va de la synonymie à l'inclusion: Ventimila leghe sotto i laghi (P 21 I 90); Pensieri in libertà (12 XI 89); Per un pugno di marchi (E 7 III 93). Dans Dolce stil rock (E 6 IX 92) et Piccolo mondo kitsch (E 3 IX 89), c'est une synonymie toute provisoire et contextuelle qui s'instaure entre rock et nuovo, kitsch et antico, tandis que dans Via con l'onda (E 16 VIII 92, sur la planche à voile), c'est plutôt la contiguitté qui opère. 
La permutation s'exerce avec une force particulière dans les noms de villes, $c f$. Addio Milano bella (E 13 XII 92; pour Lugano de la chanson). Un intertexte qui, à cet égard, se révèle d'une productivité extraordinaire est le célèbre film de Bernardo Bertolucci: Ultimo tango a Parigi devient tour à tour U. t. a Venezia (E 26 VII 92), U. t. a Madrid (E 20 X 91) U.t. a Tor Sapienza (E 12 I 92); mais la danse (au sens très large du mot!) peut être substituée elle aussi : Ultimo reggae a Giamaica (E 4 X 92); Ultimo sesso a Bangkok (E 2 VIII 92).

La plupart des cas enregistrés, toutefois, ne se laissent pas aussi facilement réduire à des lois générales. Il semble y régner une grande hétérogénéité, derrière laquelle il est sans doute possible de percevoir quelques constantes. Une de celles-ci est que l'élément lexical substitué assure l'ancrage dans le co-texte, selon un mécanisme analogue à la référence cataphorique (le titre «explique» le co-texte: $c f$. Hoek 1981:156), tandis que la partie littérale de la citation entretient avec le co-texte une relation d'ordre «rhétorique" (cf. supra), moins évidente à la lecture; p. ex.: Viaggio al centro della droga (E 22 XII 91); Chiare, fresche, confiscate acque (E 1 II 87: problèmes de disponibilité d'eau potable); Odo i libri far festa (P 14 II 93 : sur une foire du livre où les classiques ont la part belle), etc.

Dans la partie du titre qui reproduit littéralement l'intertexte, la récurrence de certaines citations révèle un véritable engouement. Ces «parties littérales» fonctionnent comme une sorte de message codé indiquant l'esprit ou l'idée générale du co-texte. En voici quelques-unes parmi les plus fréquentes:

a) Galeotto fu [il libro e chi lo scrisse: Dante, Inferno, V, 137] : trahison, imprudence fatale, traquenard non prévu, indice révélateur: Galeotto fu il fax (E 22 XII 91, sur un fax accusateur de pratiques irrégulières); G.f. quel ticket (P 6 XI 88, sur le ticket modérateur en pharmacie); G. f. il Toulà (P 30 VIII 92: rencontres suspectes entre hommes politiques au restaurant romain «Il Toulà»).

b) In principio fu [il Verbo, Jean 1:1]: valeur primordiale accordée à la chose dont il est parlé dans le co-texte: In principio fu Gutenberg (E 10 XI 91, sur une exposition de bibles anciennes); i.p. fu Mattei (P 19 VII 92, sur la création de l'ENI par Enrico Mattei), i.p. fu l'albero (P 17 I 92, sur la «fuite vers le paradis»), i.p. fu don Giusani (E 15 XI 92, sur la communauté religieuse de Sant'Egidio), i.p. fu il Simbolo (E 15 IX 91, sur le symbolisme français en peinture).

c) Così parlò [Zaratustra: Nietzsche] : introduit avec une solennité souvent antiphrastique toutes sortes de sujets: opinions diverses, innovations technologiques, etc.: Cosi parlò il videoclip (E 11 X 87, sur les simulations graphiques); C.p. Farouk (P 26 VII 92,

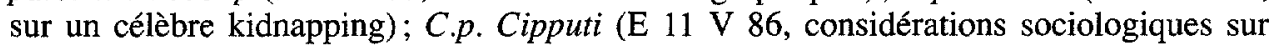
l'Italie).

d) Cogito ergo sum (Descartes) : la chose dont on parle dans le co-texte est (souvent ironiquement) donnée pour évidente, fondamentale, indispensable, inséparable de l'existence: Videocamera ergo sum (E 22 IX 91 : sur la mode des caméscopes); Video e. s. (P 17 I 02: les médias jugés par B. H. Lévy). On rencontre également la version italienne: Incesto dunque sono (E 16 X 88, sur le roman de Moravia, Il viaggio a Roma); Critico d.s. (E 18 VI 89, sur un livre d'interviews de Gianfranco Contini).

e) $[$ Hiroshima $]$ mon amour (M. Duras): préférence, mode du jour, engagement personnel: Geopolitica mon amour (E 11 X 87); Beirut m. a. (P 5 VIII 90: sur Insciallà d'Orianna Fallaci ); Tartaruga m. a. (E 6 V 90, sur la mode des tortues Ninja).

f) [Deutschland] über alles (ancien hymne national allemand, H. Hoffmann von Fallersleben): objectif à atteindre à n'importe quel prix et par n'importe quel moyen; préférence absolue accordée à...; souligne également un rapport du co-texte avec une 
réalité allemande: Rock über alles (E 15 IX 91); Italiani ü. a. (E 1 XI 92: les entreprises italiennes en Allemagne); Tatò ü. a. (E 27 IX 92: nomination, chez Mondadori, de Franco Tatò, surnommé «l'Allemand», pour ses préférences culturelles); Donatella ü. a. (P 28 II 93 : le mannequin Donatella Girombelli remporte un prix à la Modewoche de Munich).

g) Così fan tutte (Mozart-Da Ponte): universalité d'une pratique, tantôt louable (Cosi fan tutti, E, courant 1990: les commémorations mozartiennes), tantôt - et plus volontiers - illicites (Cosi facevano tutti, E 31 I 93 : les pots-de-vin).

h) Fratelli d'Italia, / L'Italia s'è desta (Goffredo Mameli). Les deux premiers vers de l'hymne national italien évoquent, avec une solennité antiphrastique, désacralisante ou désapprobatrice, tout ce qui a trait à l'Italie: Gerontocrati d'Italia (E 22 IX 91 : l'âge des politiciens); Modelle d' lt. (E 10 I 93: les «top models»); Kamerati d'It. (P 6 XII 92: les naziskins). Avec substitution du second élément: Fratelli di Germania (E 24 IV 88 : c.r. de livres sur les rapports culturels entre l'Italie et l'Allemagne). Quelques exemples pour le deuxième vers: Genova non s'è desta (P 15 XII 92: représentation au théâtre Carlo Felice de Gênes); L'Italia s'è persa (E 6 XII 87: débat sur l'identité nationale); L'It. s'è destra (P 6 XII 92 : fondation de la revue de droite L'Italia)

i) C'era una volta: l'incipit classique des contes de fées introduit, souvent avec une nuance quelque peu ironique, un co-texte où il est question d'un état de choses prestigieux, favorable, etc., et définitivement révolu: C'era una volta re Sole d'Egitto (E 28 II 93 : exposition sur Aménophis III); $C^{\prime}$. u. v. la vergine Fiat (E 7 III 93: l'entreprise turinoise était jadis au-dessus de tout soupçon...): $C^{\prime}$. u. v. il rampone (E 2 VIII 92: représentation de Moby Dick à Gênes; rampone = «harpon»); et, avec une «personnalisation» radicale et insolite: C'eravamo una volta (P 16 VIII 87: l'apogée des États-Unis vue par Gore Vidal).

D'autres intertextes favoris sont: S. Agnelli, Vestivamo alla marinara; S. Beckett, En attendant Godot [Aspettando G.] A. Fogazzaro, Piccolo mondo antico; G. Parise, Parigi o cara; G. Pascoli, $O$ Valentino vestito di nuovo; Henri IV de France, Paris vaut bien une messe [Parigi val bene una messa]; Homère, ... moi ennepe, Mousa [Cantami o Musa]; Galilée, Eppur si muove; etc.

De son côté, la partie substituée ne se limite pas à ce rôle d'ancrage sémantique dans le co-texte. Dans bien des cas s'y superpose une dimension phonologique, qui en fait un métaplasme du type calembour (Groupe $\mu$ 1970: 50-66). On ne manque pas d'exploiter toutes les formes de l'homonymie, comme le sait fort bien l'actuel (1993) premier ministre: Ha peccato, quindi è Amato (E 27 XII 92). L'homonymie peut aller jusqu'à l'holorimie du titre, tantôt presque parfaite ( $L$ a bella è la bestia, E 17 XI 91 : un calendrier avec des filles travesties en bêtes), tantôt plus approximative (Silvio, ci smembri ancora (E 14 VI 92: «démembrement» de la télévision par Berlusconi : < Leopardi, Silvia, rimembri ancora, «A Silvia», Canti, XXI). Par ailleurs, il règne ici une profusion de rimes plus ou moins riches (Ventimila beghe sotto il mare, $<V$. leghe s.il $m$., J. Verne: archéologie sous-marine; beghe $=$ «disputes»), de paragrammes (Paolo il calmo, E 13 IX 92:<P. il caldo, Brancati: interview du directeur du Corriere, Paolo Mieli; Per chi suona la carampana, E 31 I 93 : P. ch. s. la campana, Hemingway: la rivale de lady Diana: carampana $=$ «vieille sorcière»), de métagraphes (De bello laico, E 26 I 86: < De b. Gallico, César: polémiques sur «l'heure de religion»; Giacomo l' Iliadista, P 19 V 91 : < G. l'idealista, Lattuada: Giacomo Casanova traducteur d'Homère), d'enchaînements (Histoire d'Ostrica, P 31 I $93:<H$. d'O, Réage: culture d'huîtres chez les Romains), allitérations, dans le sens de «rimes initiales» (I promessi spot, E 26 X 86: < pr. sposi, Manzoni: budget de la publicité télévisée; On the Rome, E 4 VI $89:<$ On the road, Kerouac: présentation d'un jeune écrivain romain). Dans d'autres cas, on se borne à reproduire le schéma accentuel de l'original (Immunità vo cercando, E 21 VI 92: < Libertà v.c., Dante: sur les pots-de-vin; Versetti poetici, < V. satanici, 
S. Rushdie: le commentaire d'Averroës sur la Poétique d'Aristote). (cf. Groupe $\mu 1970$ : 57 58, 65; Guiraud 1979: 7, 13-14, 31-32; Valesio 1967: 27).

Ces métaplasmes apparaissent, en quelque sorte, comme des têtes de Janus : comme n'importe quelle partie substituée, ils assurent le raccord avec le co-texte; mais, de surcroît, leur aspect phonique renvoie à la partie de l'intertexte qu'ils transforment.

Cette prédominance indiscutable du type «non littéral, fermé» ne peut que correspondre à une préférence très marquée des lecteurs pour ce genre de titres, qui révèlent expressément leur double référence, au co-texte et à l'intertexte. Par leur jeu - tantôt ingénieux, tantôt un peu pesant - sur l'explicite et l'implicite, le permanent et le changeant, ils combinent de façon visible la dénotation et la connotation, leur rôle d'identification et leurs potentialités intertextuelles et littéraires (au sens large). Ce sont aussi les titres de cette catégorie qui accumulent le plus de facteurs différents - sémantiques, logiques, phonologiques, rythmiques - aptes à se signaler à l'attention du lecteur.

Voici un tableau des provenances des titres de notre corpus, classées par ordre décroissant :

28: Bible

17 : Dante

11 : G. Mameli (hymne national italien)

10: Shakespeare

8: Descartes

7 : Leopardi, Manzoni, Nietzsche, prières et formules rituelles, E. Scola

6 : U. Eco, F. Fellini

5 : B. Bertolucci, César, Henri IV, Galilée, Homère, K. Marx, C. Pavese, L. Sciascia, W. S. Van Dyke

4 : S. Agnelli, S. Beckett, «Blanche-Neige», A. Fogazzaro, E. Hemingway, H. Hoffmann von Fallersleben (hymne national allemand), «Il était une fois», I. Kershner, La Belle et la Bête, $J$. LeCarré, S. Leone, W. A. Mozart / L. Da Ponte, G. Parise, G. Pascoli, Pétrarque, M. Proust, R. L. Stevenson, G. Tornatore

3 : E. Albee, H. C. Andersen, C. Aznavour (ch), H. Böll, «Borghesia»(ch), Constantin, $G$. Damiano, M. Duras, F. S. Fitzgerald, N. Ginzburg, J.-L. Godard, C. Goldoni, T. Hackford, J. Kerouac, C. Levi, M. Mitchell, L. Pirandello, G. Verdi, J. Verne, T. Williams

2 : «Addio Lugano bella»(ch.), Archimède, S. Benelli, I. Calvino, G. Carducci, L. Carroll, F. Cristaldi, M. Curtiz, V. De Sica, D. Diderot, G. García Marquez, Gino \& Michele, W. Goethe, G. Jacopetti, N. Jewison, R. Kramer, G. Ledda, S. Leone, P. Levi, F. T. Marinetti, J. Milton, F. Molnar, E. Morante, G. Orwell, S. Pellico, P. Réage, F. Rosi, S. Rushdie, F. Sagan, M. Tobino, J. R. R. Tolkien, C. Vanzina, L. Visconti, E. Vittorini, T. Young

1 : V. Alfieri, W. Allen, C. Angiolieri, Anonimo Romano, M. A. Antonioni, Archimède, $I$. Bergman, R. Bolt, V. Brancati, B. Brecht, F. Brusati, A. Burgess, J. Cain, R. Calasso, P. Calderón, F. Capra, C. Cavour, L.-F. Céline, V. Cerami, C. Chaplin, A. Conan Doyle, F. Cooper, E. De Filippo, S. Eizenstein, D. Fo, E. Forster, François d'Assise, W. Friedkin, Fruttero e Lucentini, C. E. Gadda, «Gloria in excelsis» (rite), M. Gray, J. Hackford, E. Haraucourt, V. Hugo, N. Kazantzakis, K. Kostner, A. Lattuada, «Le corbeau et le renard»(fable), A. Llewelyn, G. Leroux-R. Julian, «L'important, c'est la rose» (ch.), Louis XV, C. Malaparte, H. Marcuse, Molière, R. Musil, Oshima, A. Pakula, P. P. Pasolini, E. Petri, Philippe VI de France, «Populorum progressio» (encyclique), V. Pratolini, S. Quasimodo, R. Queneau, L. Ravera, G. Rodari, R. Rossellini, G. Rossini, L. Sailer (ch.), «Salve regina» (rite), S. Soderberg, M. Soldati, S. Spielberg, H. M. Stanley, J. Steinbeck, A. Tabucchi, V. et P. Taviani, Tirso de Molina, G. Ungaretti, D. F. Zannuck

TOTAL : $439+11$ (douteux) $=450$

ch.= chanson. En italiques, les réalisateurs de films ou les auteurs d'œuvres littéraires dont il existe une importante adaptation cinématographique. 
On ne sera pas étonné de constater qu'une grosse proportion de nos titres (au moins un quart) a quelque rapport avec le cinéma, qu'il s'agisse d'œuvres cinématographiques originales ou de livres portés à l'écran après avoir été diffusés par l'édition. Il faut se faire une raison: le cinéma est un des grands médias de notre siècle, redoutable concurrent de la lecture - l'a-t-on assez répété -, mais aussi voie d'accès privilégiée à toute forme de littérature fictionnelle: qui dit James Bond pense plutôt aux films avec Sean Connery qu'aux romans de Ian Fleming; et qui, avant le film de Kubrick, connaissait l'existence du roman Barry Lyndon de Thackeray? Il semble raisonnable de supposer que, si un livre a été adapté pour le cinéma, c'est cette dernière version qui aura le plus d'impact et sera le plus volontiers citée. On se gardera toutefois de généraliser: Il Gattopardo de Giuseppe Tomasi di Lampedusa (1958), Padre padrone de Gavino Ledda (1975) et Un borghese piccolo piccolo de Vincenzo Cerami (1976) ont connu un beau succès éditorial avant d'être adaptés au cinéma par Luchino Visconti (1963), les frères Taviani (1977) et Mario Monicelli (1979). Il en est de même pour Via col vento (Gone with the Wind, Autant en emporte le vent): le film de Selznick (1939) est certes un monument du cinéma, mais le roman de Margaret Mitchell a été un best-seller en Italie bien avant qu'on n'y découvre le film: bien plus, la traduction d'Ada Salvatore et d'Enrico Piceni (1937) est parmi ces livres qui, par leur large diffusion, ont pu contribuer à imprimer une certaine évolution à l'italien moderne (De Mauro 1963: 351-352) ${ }^{5}$. Certains de nos titres traduisent d'ailleurs de manière tangible l'influence simultanée du cinéma et de la lecture: on trouve des références tant à $I l$ contesto de Sciascia (1971) qu'à Cadaveri eccellenti, le film qui en a été tiré par Francesco Rosi (1976).

$\mathrm{Si}$, à l'intérieur de notre corpus, le cinéma se signale par sa présence massive dans le processus de réminiscence littéraire, on y trouve d'autres formes tout aussi particulières de «littérature»: ainsi, on remarquera la prédilection pour le conte de fées ( $c f$. C'era una volta; Chi è la più bella del reame?), les «paroles historiques» (cf. Eppur si muove; Veni, vidi, vici), les chansons, anciennes ( $c f$. Addio Lugano bella ; Faccetta nera, bell'Abissinia) ou modernes ( $c f$. Que c'est triste Venise), les hymnes nationaux (italien et allemand), les prières et les formules rituelles du catholicisme romain (cf. Gloria in excelsis Deo; Salve regina): autant de productions textuelles situées aux confins de la littérature au sens restreint, mais faisant partie d'une sorte de «réservoir de phrases toutes faites» collectif, de substrat commun, au même titre que les dictons, les proverbes, etc.

Les réminiscences bibliques y ont aussi leur place: bien plus, avec 28 attestations, la Bible apparaît comme le texte le plus cité, ce qui n'a rien de surprenant dans la réalité historique et culturelle de l'Italie. On observera la forte présences de certains passages, appartenant presque tous au Nouveau Testament (l'exception la plus notable est «Tu enfanteras dans la douleur», Gen. 3:16). Un tiers de ces cas se réfère à Jean 1:1 («Au commencement était le Verbe»); d'autres versets favoris sont: «Laissez venir à moi les petits enfants» (Mat. 19:14; Marc 10:14; Luc 18:16) et «L'homme ne vit pas que de pain»(vétérotestamentaire, Deut. 8:3, mais repris dans Mat. 4:4 et Luc 4:4). On ne perdra pas de vue que certains de ces passages sont devenus des formules rituelles (p. ex. l'oraison dominicale, citée 3 fois; et également le début de l'Évangile selon Jean) : si bien qu'il est peu probable que nous ayons affaire à des souvenirs de lecture, même indirects.

$\mathrm{Au}$-delà de ces faits littéraires périphériques, il y a néanmoins dans notre corpus un «noyau dur» de références qui ne peuvent être ramenées qu'à la littérature au sens plus restrictif : poèmes narratifs, poésie lyrique, théâtre, roman... On ne manquera pas d'être frappé par la présence massive des «classiques», et avant tout de Dante qui, cité ou évoqué 17 fois, constitue le deuxième intertexte par ordre d'importance (ne fut-il pas une époque où «les Italiens connaissaient leur Dante par cœur»?); mais Pétrarque et Manzoni, Goldoni et Pellico, Leopardi et Carducci, Pascoli et Fogazzaro se classent de façon très 
honorable; même François d'Assise et Cecco Angiolieri ne sont pas absents. Il n'est pas douteux que cette «orientation classique» soit une empreinte laissée par l'école. C'est à elle aussi qu'on fera remonter les souvenirs d'auteurs grecs et latins (Homère, César), de philosophes (Descartes, Nietzsche) et de certains classiques étrangers (Shakespeare, Milton, Goethe, etc.). Il se peut que ce poids de l'éducation scolaire soit quelque chose d'assez spécifique dans la culture italienne.

D'autres secteurs de la littérature ne sont pas pour autant délaissés. Parmi les auteurs italiens de la première moitié du siècle, Pirandello et Marinetti bénéficient d'un certain succès, de même que, chez les étrangers, Proust, Fitzgerald, Orwell, Hemingway, Tenessee Williams, Margaret Mitchell (on fait abstraction, pour le moment, des interférences avec le cinéma). Des décennies suivantes, on retient surtout, chez les Italiens, Pavese, Carlo Levi, Primo Levi et Vittorini ; hors d'Italie, Eduard Albee, Heinrich Böll, Jack Kerouac, Marguerite Duras, Françoise Sagan. Même des auvres tout à fait contemporaines peuvent avoir un impact suffisant pour déclencher des réminiscences: ainsi Calvino, Susanna Agnelli, Goffredo Parise, Leonardo Sciascia, Antonio Tabucchi, Natalia Ginzburg, Gabriel García Marquez, etc.: jusqu'au livre de Gino \& Michele, Anche le formiche nel loro piccolo s'incazzano, publié il y a trois ans à peine. À cet égard, Umberto Eco constitue le cas rare et enviable d'un auteur contemporain souvent cité, autant que Fellini et plus souvent que Pascoli et Galilée! Pour d'autres cuvres, tels les Versets sataniques de Salman Rushdie, il est vraisemblable que la relative faveur qu'elles rencontrent dans le corpus est due à un succès de scandale, comme ce fut jadis le cas d'Histoire d'O et de Porci con le ali. On notera aussi la présence occasionnelle de curieux «titres-valises» : ainsi Dalla Russia con le ali, hybridation de Dalla Russia con amore (From Russia with Love, Bons baisers de Russie) de Terence Young (d'après I. Fleming) et de Porci con le ali de Lidia Ravera. D'autres titres laissent planer une incertitude quant à leur intertexte : ainsi, Sodoma e Berlino (E 10 II 91) peut renvoyer à la Recherche de Proust, mais aussi au livre de Malaparte $S$. e Gomorra (1931), ou bien encore, génériquement, à l'épisode biblique.

Il est à remarquer que, dans cette «bibliothèque idéale» qui sert de point de référence aux titres, il n' y a pas vraiment de barrières linguistiques et nationales. S'il est vrai que la place importante occupée par les classiques italiens n'est concevable qu'en Italie, on ne peut pas toutefois parler d'une prédominance de la littérature italienne (qui ne représente que 43,5\% des cas): Descartes et Homère, Nietzsche et César, Shakespeare et Samuel Beckett ne sont guère moins cités; et la popularité d'un Eco ou d'un Galilée n'est probablement pas moindre hors des frontières de l'Italie. Tous font partie intégrante de ce bien culturel commun européen, où les découpages n'ont qu'une valeur relative. Le caractère résolument «transeuropéen» de notre corpus se marque parfois jusque dans le recours à une autre langue, comme On the road, Histoire d'O, [Hiroshima] mon amour; D'autre part, on trouve peu ou point de références aux grandes littératures en langues non européennes (on exceptera le film japonais L'Empire des sens et, hors de ce corpus 1993, les Mille et une nuits).

Il serait sans doute instructif de comparer nos résultats de maintenant à ceux de notre enquête de 1981 (Bastiaensen 1982 a) : ce travail demanderait toutefois un peu plus d'espace et devrait tenir compte de la différence quantitative entre les deux corpus (325 contre 450). Ces réserves faites, on peut constater que le «peloton de tête» était déjà alors occupé par la Bible (dans une proportion plus élevée), Dante, Leopardi, Nietzsche et Shakespeare; Manzoni et Descartes y apparaissaient avec une fréquence moindre, tandis que Pirandello, Carlo Levi et Salvatore Quasimodo semblent avoir eu alors un succès bien plus grand que maintenant. On y trouvait aussi, parmi les auteurs cités plus de deux fois, l'Arioste, Beccaria, Nabokov et Verga, qui ont disparu de notre corpus actuel; mais 
celui-ci accueille de nouveaux venus comme Umberto Eco, Susanna Agnelli, Jack Kerouac - outre, évidemment, Salman Rushdie. On voit que, s'il y a un certain nombre de différences - explicables par les modalités de transmission et de connaissance, les goûts et les modes, l'évolution dans l'âge des lecteurs, - il reste néanmoins un noyau commun, apparemment peu affecté par le changement et le passage des années.

\section{QUE FAIRE ?}

Le mécanisme particulier d'intertextualité que nous avons envisagé n'est pas sans entraîner quelques conséquences sur le fonctionnement même du message. La compréhension d'un texte dans sa totalité est aussi celle de son titre, en raison de leur dépendance contextuelle mutuelle (Hoek 1981 : 152). Or certains titres peuvent à première vue paraître bien mystérieux et ne montrer en rien ce rapport au co-texte: que vient faire le Galehaut des romans bretons dans l'administration sanitaire ou l'expédition de fax? Pourquoi s'adresse-t-on à un bœuf en disant qu'il est «pieux»? À quoi bon introduire n'importe quel sujet par «Ainsi parla» ou «Chante-moi»? Pourquoi ces archaïsmes, inversions, élisions, termes dialectaux, étrangers, latins? Le problème ne se limite bien sûr pas au seul titre: on songera avec Žarko Muljačić (1971 : 98-99) à l'adjectif vispoteresoide, forgé par C. E. Gadda sur la vispa Teresa (vispo, «vif»), personnage d'une chansonnette enfantine de Luigi Sailer (1825-1885). Gageons que même un extraterrestre très doué, dont le cerveau aurait emmagasiné toutes les grammaires et tous les dictionnaires, y perdrait quelquefois son latin (si l'on ose dire...). Et quiconque enseigne à des étudiants terriens sait fort bien que des traductions comme «Le galérien, ce fut un livre» (Galeotto fu il libro) n'appartiennent pas au domaine de l'imagination.

En fait, il y a là toute une information à acquérir en même temps que les niveaux les plus avancés de la langue étudiée. Même si cette information intertextuelle peut rester assez générique - comme elle l'est souvent pour le lecteur moyen - elle n'en est pas moins indispensable pour la compréhension de textes aussi courants que les articles de presse.

Oublions un instant que l'Italie est proche et adoptons le point de vue de Sirius. Si parfaite que soit la connaissance linguistique, on ne peut vraiment lire un texte sans s'être familiarisé - ce devrait être une lapalissade - avec le culture dont il est issu : culture au sens le plus large, incluant le «non-dit» qui sous-tend les mots et les phrases et leur donne une dimension de sens qui les dépasse. Comment pourrait-on, par exemple, non pas être Persan, mais aborder la poésie persane classique sans s'être d'abord informé sur des stylèmes comme «le supérieur du couvent des mages» et «la boucle de cheveux de la bienaimée», qui ne signifient pas ce qu'ils paraissent désigner (cf. Bausani 1968: 138-181)? Toute approche d'un texte suppose une «compétence communicative» ou «culturelle» (cf. Hymes 1971, in Sandulescu 1986:613).

Mais revenons au domaine italien (où l'intertextualité ne se limite pas, rappelonsle, à la seule littérature italienne). Les dictionnaires sont, en général, de peu d'utilité pour dépister les réminiscences littéraires, encore qu'on puisse espérer en trouver parmi les innombrables exemples du Battaglia; mais, symptomatiquement, les dictionnaires de néologismes en enregistrent quelques-unes ( $c f$. Cortelazzo-Cardinale 1989: 10, 89, 115, 204; Bencini-Citernesi 1992: 60,70,71, 78, 151, 226, 325, etc.). Pour les références plus anciennes, le recours aux dictionnaires de citations peut donner de bons résultats: on songera, pour l'Italie, à ceux de Giuseppina Fumagalli et de F. Palazzi - S. Spaventa Filippi. Par ailleurs, il ne serait ni irréalisable ni inutile de constituer un répertoire éventuellement informatisé - de ces titres littéraires d'articles de presse, comprenant les originaux et leurs transformations : un tel répertoire nécessiterait sans doute, autour d'un noyau permanent ( $c f$. supra), de fréquents compléments et mises à jour. 
Surtout, il est indispensable que l'enseignement, et en particulier l'enseignement de la traduction, tienne compte de ces implications intertextuelles, dans la mesure où elles ont une forte incidence sur la compréhension même du texte. C'est dire l'importance fondamentale des cours à vocation culturelle, qui s'intitulent «civilisation», «littérature», «institutions», et qui ne sont que trop souvent considérés comme périphériques ou marginaux. Quant aux cours de traduction stricto sensu, des titres de ce type permettent aux étudiants comme aux enseignants d'exercer une belle créativité verbale, dont voici quelques spécimens sortis de nos leçons des années précédentes: Galeotto fu il ticket: «C'est la faute à Voltaire, c'est la faute au ticket»; Vaghe stelle dell' Orsa (Leopardi; intitule un chapitre d'un livre de Cesare Musatti sur la psychanalyse $\left.{ }^{6}\right)$ : «Cette obscure clarté qui tombe des étoiles» (Corneille); Gerontocrati d'Italia: «Allons, vieillards de la patrie !»; Europa : chi era costei? "Europe, unique objet de mon ressentiment»; etc.

\section{CONCLUSION}

L'《intertextualité titulaire» évoquée dans ces pages n'est sans doute pas un phénomène limité à l'Italie. Il n'empêche que sa présence y est fort tangible, et qu'elle s'impose avec plus de force encore à qui aborde les textes d'un point de vue contrastif. L'approche d'un texte et la volonté de le comprendre dans tous ses aspects amènent inévitablement le lecteur, et le lecteur privilégié qu'est le traducteur, à accorder une attention toute particulière aux titres de ce genre, à étudier leur motivation par rapport au co-texte, à en démonter les mécanismes rhétoriques, à identifier les tendances qui président aux modifications qu'ils subissent, à en retracer l'origine et le cheminement. Un tel travail, entrepris aussi dans l'espoir de résoudre certaines questions précises de compréhension et de traduction et de contribuer à améliorer l'information dans ce domaine précis, aboutit au tableau d'une certaine culture littéraire - celle que sont censés posséder les lecteurs d'hebdomadaires d'information connotés comme socialement et culturellement «élevés». Culture littéraire d'une physionomie bien particulière, où les romans policiers voisinent avec le bagage classique de l'école, Tarzan avec les chefs-d'œuvre poétiques, la bande dessinée avec la philosophie... Culture littéraire banalisée et «massifiée», si l'on veut, et qui est loin d'impliquer toujours un contact direct avec les œuvres. Mais, comme pour les princes de Sérendib, la quête est peut-être plus enrichissante que l'objet recherché.

Notes

1. Auxquels ils ne sont en aucun cas limités: on songera p. ex. à : Faulkner, The Sound and the Fury < Macbeth, V, 5; Steinbeck, The Grapes of Wrath < Apocalypse 14:16; Visconti, Vaghe stelle dell' Orsa < Leopardi; etc.

2. Ainsi, nous trouvons, dans Le Vif-L'Express du 12-18 février 1993: Onze hommes colère; Au nom de tous les siens; Haro sur le goupil!; Un seul être vous manque...; Ma vie, qu'est-ce qu'elle a, ma vie ?; Gorbatchev: ce que je croyais.

3. Corriere della Sera, courant 1990 , retenu pour sa valeur exemplaire, non comptabilisé dans notre corpus.

4. Cf. Hoek 1981: 18: «Le terme 'co-texte' est emprunté à Petöfi [...] pour indiquer ici l'ensemble de phrases qui suivent ou qui devraient suivre le(s) titre(s) mentionné(s) à la page de titre. Le co-texte est ainsi l'équivalent du texte dépourvu de son titre. Le terme 'contexte' désigne l'ensemble des facteurs verbaux (intertextuels) et non verbaux (situationnels) qui jouent dans le procès de communication et qui se trouvent hors du texte en question.»

5. En 1947, Via col vento est le troisième des livres les plus lus en Italie (après Les fiancés de Manzoni et La mousson de Bromfield); en 1949, il prend la première place (une influence du film n'est pas douteuse), mais, en 1954, il disparait du peloton des vingt premiers.

6. C. Musatti, Questa notte ho fatto un sogno; mémoire de licence de M. Vincent Van Halteren (1990-1991) 


\section{RÉFÉRENCES}

BASTIAENSEN, Michel (1982a) : «Sostrato letterario e stampa periodica», Marc Dominicy (Éd.), Université Libre de Bruxelles. Actes de la deuxième journée des romanistes, 8 novembre 1981, Bruxelles, pp. 74-86.

BASTIAENSEN, Michel (1982 b) : «Littérature, presse et titres», Enjeux. Revue de didactique du français, 1 (Namur), pp. 68-85.

BAUSANI, Alessandro (1968): La letteratura neopersiana, Antonio Pagliaro et Alessandro Bausani (Éds.), La letteratura persiana, Firenze, Sansoni, nouvelle édition mise à jour.

BENCINI, Andrea et Eugenia CITERNESI (1992): Parole degli anni Novanta, Firenze, Le Monnier; «Materiali e ricerche per il Devoto-Oli», a C. di Gian Carlo Oli.

CORTELAZZO, Manlio et Ugo CARDINALE (1989): Dizionario di parole nuove, 1964-1987, Torino, Loescher.

DE MAURO, Tullio (1963) : Storia linguistica dell' Italia unita, Bari, Laterza.

FUMAGALLI, Giuseppina (1934): Chi l' ha detto ?, Milano, Hoepli (réimpr. 1951).

GENETTE, Gérard (1987) : Seuils, Paris, Seuil.

Groupe $\mu$ : DUBOIS, J., EDELINE, F., KLINKENBERG, J. M., MINGUET, P., PIRE, E. et H. TRINON (1970) : Rhétorique générale, Paris, Larousse.

GUIRAUD, Pierre (1979): Les jeux de mots, Paris, PUF, $2^{\circ}$ éd.

HALLYN, Fernand et Georges JACQUES (1987): «Aspects du paratexte», Maurice Delcroix et Fernand Hallyn, Méthodes du texte, Gembloux, Duculot, pp. 202-215.

HENRY, Albert (1984) : Métonymie et métaphore, Bruxelles, Palais des Académies («Mémoires de la Classe de Lettres, collection in- $8^{\circ} \gg, 2^{2}$ série, vol. LXVI / 2 ), édition revue, ( ${ }^{\text {re }}$ éd. : Paris, Klincksieck, 1971).

HOEK, Leo H. (1981) : La marque du titre. Dispositif sémiotique d'une pratique textuelle, La Haye, Mouton.

HYMES, Dell (1971): «On Communicative Competence», J. B. Pride et J. B. Holmes, Sociolinguistics, London, Penguin, pp. 269-294.

MANDELBROT, Benoit (1954) : «Structure formelle des textes et communication. Deux études», Word, X/1, pp. 1-27.

MEDICI, Mario et Sonia CAPELLUZZO SPRINGOLO (1991) : Il titolo del film nella lingua comune, Roma, Bulzoni ( $\ll$ Biblioteca cinematografica e dei mass-media», n. 32).

MULJAČČC, Žarko (1971): Introduzione allo studio della lingua italiana, Torino, Einaudi.

PALAZZI, F. et S. SPAVENTA FILIPPI (1950): Il libro dei mille savi, Milano, Hoepli ( $2^{\mathrm{e}}$ éd.).

SANDULESCU, C. George (1986): «The Joycean Archetype», Daniela Dalla Valle, Manierismo e letteratura. Atti del congresso internazionale, Torino, 12-15 ottobre 1983, Torino, Meynier, pp. 607-628.

VALESIO, Paolo (1967) : Strutture dell'allitterazione, Bologna, Zanichelli.

VALESIO, Paolo (1967) : Strutture dell' allitterazione, Bologna, Zanichelli. 